\title{
Solvation of $\mathrm{NaPF}_{6}$ in diglyme solution for battery electrolytes
}

\author{
Anders C. S. Jensen ${ }^{\star a}, \mathrm{~b}$, Heather $A u^{b}$, Sabrina Gärtner ${ }^{c}$, Maria-Magdalena Titirici ${ }^{\mathrm{b}}$ and Alan J. Drew
}

[a] Dr. A. C. S. Jensen*, Prof. A. J. Drew

School of Physics and Astronomy

Queen Mary University of London

Mile End Road, London, E1 4NS, United Kingdom

E-mail: a.jensen@qmul.ac.uk

[b] Dr A. C. S. Jensen, Dr. H. Au, Prof. M.-M. Titirici

Department of Chemical Engineering

Imperial College London

London, SW7 2AZ, United Kingdom

[c] Dr S. Gärtner

ISIS Neutron and Muon Source

STFC Rutherford Appleton Laboratory

Chilton, Oxfordshire OX11 OQX, United Kingdom

Supporting information for this article is given via a link at the end of the document.

\begin{abstract}
Glymes present a promising new group of electrolyte solvents for sodium ion batteries. Not only do they have excellent electrolyte solvent properties but they also enable the intercalation of sodium into graphite as sodium-glyme complexes, a reaction which is not possible for sodium in conventional electrolyte solvents. However, little is known about the solution structure of these complexes, especially for sodium salts, and why glymes enable this process while other commonly used electrolyte solvents do not. Here, a combination of neutron total scattering and empirical potential structure refinement was used to characterize the solvent structure around the ions, for a $\mathrm{NaPF}_{6}$ solution in diglyme. This showed that $82 \%$ of the sodium ions are bound as $\mathrm{Na}^{+}$(diglyme) ${ }_{2}$ complexes, the conformation needed for intercalation into graphite, with the rest forming various contact ion pairs. The model also showed that very weak hydrogen bonding interactions exist between the anion and the diglyme molecules.
\end{abstract}

\section{Introduction}

Glymes make up an interesting group of electrolyte solvents for battery technologies, offering high ionic conductivity, a wide operating potential window and high thermal stability. ${ }^{[1]}$ However studies have shown a link between diglyme and testicular toxicity, and as such it should be handled with care. ${ }^{[2]}$ Additionally, glymes have been shown to work for a wide range of battery technologies including lithium, ${ }^{[1,3]}$ magnesium ${ }^{[4]}$ and sodium ion batteries. ${ }^{[3 a, 5]}$ Diglyme has shown great promise in sodium ion batteries (SIB) with similar performance to carbonate electrolytes but with a wider voltage range and reduced capacity loss in the first cycle; indeed, inNa $\mathrm{V}_{2}\left(\mathrm{PO}_{4}\right)_{3}$ cathodes no initial loss is observed. [5b, 6] Excellent performance has also been reported for layered oxide cathodes. ${ }^{[7]}$

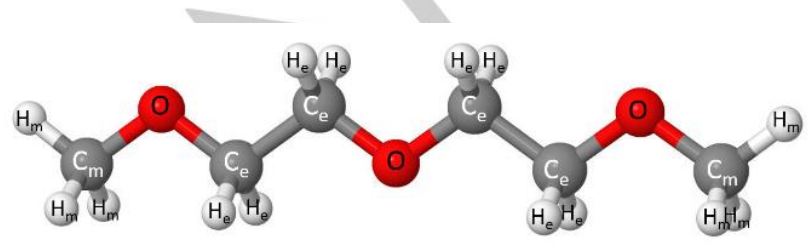

Figure 1: Molecular structure of diglyme $(n=2)$, with the subscripts $\mathrm{m}$ and e denoting the end groups and the chain groups, respectively.
Mixed performance for glymes has been reported for hard carbon anodes, ${ }^{[6,8]}$ but this could be a result of the large difference in surface reactivity of different hard carbon anodes. ${ }^{[9]}$ Good performance was reported for both carbon nanofibers and graphite..$^{[5,10]}$ In graphite anodes, diglyme allows sodium to intercalate into graphite as e.g. $\mathrm{Na}^{+}$(diglyme) ${ }_{2}$ complexes, increasing the capacity from $12-35 \mathrm{mAh} / \mathrm{g}$ in conventional carbonate solvents to $110 \mathrm{mAh} / \mathrm{g}$ in diglyme. ${ }^{[5 \mathrm{~b}, 11]}$ Longer glymes with the general structure $\mathrm{CH}_{3}-\mathrm{O}\left(-\mathrm{CH}_{2}-\mathrm{CH}_{2}-\mathrm{O}\right)_{n}-\mathrm{CH}_{3}$ (Figure 1) or crown ethers with cyclic structure have also been shown to work well for graphite anodes. The linear glymes with $n=1-4$ have been shown to give good capacity in SIB with graphite anodes at room temperature, while longer chained and cyclic glymes become electrochemically active only at elevated temperatures. ${ }^{[3 a, 5]}$ At all temperatures, diglyme $(n=2)$, shown in Figure 1 , has the highest capacity and rate performance of the studied glymes. ${ }^{[5 a]}$ The graphite anode in combination with a layered oxide cathode has shown reasonable cycle stability but limited capacity at $\sim 100$ $\mathrm{mAh} / \mathrm{g}$ at $0.2 \mathrm{C} \cdot{ }^{[12]}$ Soft carbon, thermally expanded graphite and oxidized graphite show similar performance to graphite, implying that the high degree of order in pyrolytic graphite is not necessary for the reversible intercalation of $\mathrm{Na}^{+}$(diglyme) ${ }_{2}{ }^{[13]}$

Several lithium, sodium and potassium salts form crystalline compounds with glyme molecules at room temperature, where the structure can be easily examined by X-ray diffraction (XRD) techniques. ${ }^{[14]}$ This has shown three characteristic local structures that the cations can adopt, influenced by both the cation, anion and type of glyme present in the crystal. In the case of pentaglyme-coordinated lithium bis(trifluoromethanesulfonyl)imide (Li(G5)TFSI), the cation is fully coordinated by the pentaglyme (G5) molecule and separated from the anion forming a solvent separated ion pair (SSIP). ${ }^{[14-15]}$ In contrast, the cation in $\mathrm{Na}^{+}(\mathrm{G} 5)$ TFSI is coordinated by both G5 and a single oxygen from the TFSI anion, forming a contact ion pair (CIP) ${ }^{[14]}$ CIP can be further categorised based on the number of coordinating atoms provided by the anion, with examples of monodentate binding (CIP-1) in $\mathrm{Na}^{+}(\mathrm{G} 5)$ TFSI and bidentate binding in Li(G3)TFSI (CIPII). ${ }^{[14]}$ If strong interactions between the cation and anion are present, aggregation (AGG) of the ions can occur where the anion is coordinating several cations. ${ }^{[14,16]}$

Translating this understanding to the liquid phase poses a significant challenge, as the level of structural detail provided by $\mathrm{XRD}$ is not achievable for a liquid. However, this understanding is important as the interactions in solution dictate the properties of the electrolyte. This is especially evident in the stability of the solvent as uncoordinated glyme molecules exhibit a lower 

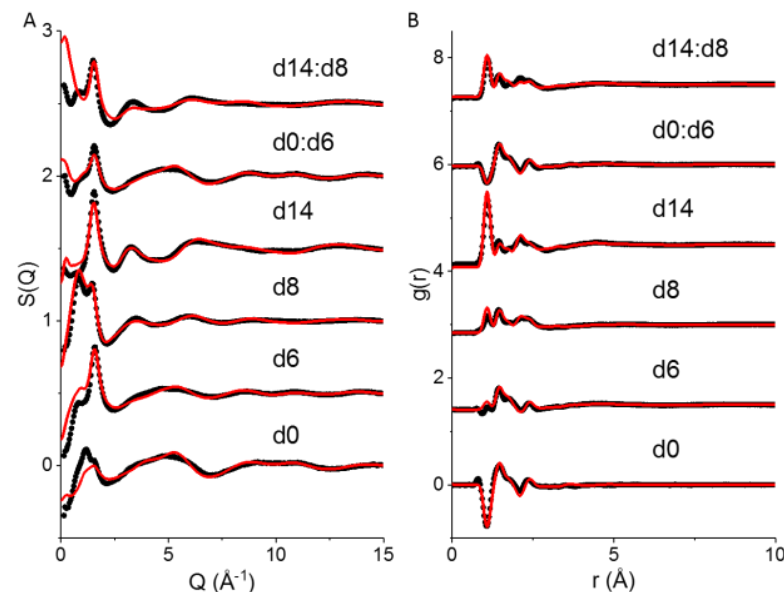

Figure 2: EPSR simulated (red) and measured (black) $S(Q)(A)$ and the derived $g(r)(B)$ of the $1 \mathrm{M} \mathrm{NaPF}_{6}$ solution with the different deuterated compounds. The same results for the pure diglyme solution are shown in Figure S4.

oxidation potential than glyme participating in cation coordination, due to shift in the HOMO level. ${ }^{[6,14,17]}$ Similar shifts are observed for the reduction potential, which vary from $-0.07 \mathrm{~V}$ to $-1.21 \mathrm{~V}$ depending on the bond and coordination environment. ${ }^{[6]}$ This also affects diffusive properties of the electrolyte with aggregation associated with lower ionic conductivity. ${ }^{[18]}$

In the absence of XRD techniques, solution studies have relied on vibrational spectroscopy techniques, such as Raman and Fourier Transform Infra-Red (FTIR), combined with theoretical modelling. Here the local coordination is examined by the shift in frequency of specific vibrations associated with either the solvent molecule or the anion, which are highly sensitive to directly coordinating atoms or molecules. ${ }^{[19]}$ Several broad studies of sodium electrolytes have showed that sodium ions generally exhibit fewer ion-ion interactions compared to lithium ions, resulting in faster kinetics. ${ }^{[19 d, 20]}$

For sodium triflate (NaOTf) in various glymes, FTIR and ab initio molecular dynamics (AIMD) showed that SSIP, CIP and AGG local structures are all present in concentrations from $0.5-2 \mathrm{M}$, with a higher fraction of CIPs and AGG at elevated concentration; similar results were shown for sodium bis(fluorosulfonyl)imide (NaFSI) ${ }^{\left[19 c,{ }^{21]}\right.}$ However, as shown for lithium nitrate $\left(\mathrm{LiNO}_{3}\right)$ and LiTFSI dissolved in tetraglyme at high concentration, the different anion can have a large impact on the probability of forming ion pairs. ${ }^{[22]}$

For sodium hexafluorophosphate $\left(\mathrm{NaPF}_{6}\right)$, computational studies have suggested that the anion should favour formation of bidentate and tridentate CIPs. ${ }^{[19 a, 19 b]}$ Raman spectroscopy has suggested the presence of both SSIP and CIPs with increasing CIPs at higher concentration. ${ }^{[23]}$ The presence of CIPs for $\mathrm{LiPF}_{6}$ in diglyme has also been reported. ${ }^{[24]}$

Using neutron diffraction with isotope substitution, we show here that $\mathrm{NaPF}_{6}$ in diglyme forms mainly $\mathrm{Na}^{+}$(diglyme) $)_{2}$ complexes, with the anion separated from the cation by $\sim 6 \AA$, forming SSIPs. Additionally, minor populations of other species are observed with less than $\sim 20 \%$ of the sodium forming CIPs. The most commonly found $\mathrm{Na}^{+}$(diglyme) ${ }_{2}$ complex is similar to the description of the sodium diglyme complexes intercalated into graphite by Goktas et al. ${ }^{[5 a]}$

\section{Empirical potential structure refinement}

Empirical potential structural refinement (EPSR ${ }^{[25]}$ has previously been applied to several liquid[22, 26] and amorphous systems ${ }^{[27]}$ to analyse the short and medium range structure from total scattering neutron and X-ray data. EPSR uses a Monte Carlo routine to generate an initial structure based on an experimentally
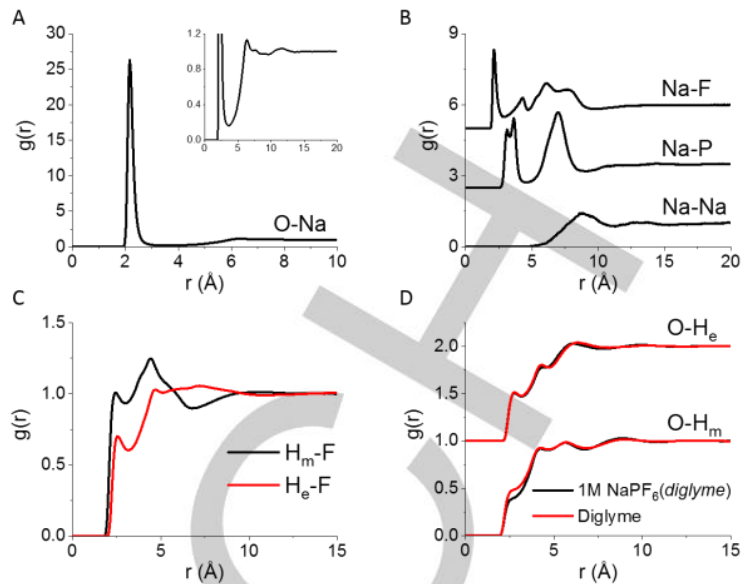

Figure 3: PPDFs extracted from the EPSR model showing the PPDF related to A) $\mathrm{Na}-\mathrm{O}$ bonding (inset shows an expanded view of the long range correlations), B) ion-ion interactions, C) $\mathrm{F}-\mathrm{H}$ hydrogen bonds between $\mathrm{PF}_{6}$ - and diglyme and $\mathrm{D}$ ) hydrogen bonding between diglyme molecules. Remaining PPDF are shown in Figures S5 and S6.

determined density, composition and a reference potential. The neutron or X-ray data is introduced by estimating an empirical potential from the difference between the calculated structure factor $(S(Q))$ based on the energy minimised structure generated from the Monte Carlo routine and the experimentally determined $S(Q)$. The empirical potential is iteratively refined to give the final structure. This allows the EPSR model to go beyond the first coordination shell typically accessible by vibrational spectroscopy, similar to standard Monte Carlo simulations but refined against experimental data.

\section{Results and Discussion}

The measured neutron total scattering results for the different contrasts are shown in Figures 2 and S4. The EPSR ${ }^{[25]}$ model was energy minimized using an all-atom optimized potentials for liquid simulations (OPLS-AA) reference potential, ${ }^{[28]}$ after which the empirical potential was added and the final result was averaged from over 10,000 configurations. This showed a good agreement with the measured $S(Q)$ and the pair distribution function $(g(r))$ for both the pure diglyme and the $1 \mathrm{M} \mathrm{NaPF}_{6}$ solution.

Based on the EPSR model, the partial pair distribution functions (PPDF) were estimated and selected pairs are presented in Figure 3. The main interactions involving $\mathrm{Na}$ are with $\mathrm{O}$ from diglyme forming a direct bond with length $2.16 \AA$ (Figure $3 A$ ) and less frequently, bonds with the $\mathrm{PF}_{6}$ anion as seen by the peaks at 2.13 and $4.3 \AA$ in the Na-F PPDF and the peak at $3.3 \AA$ in the Na-P PPDF (Figure $3 B$ ). A second coordination sphere is evident in the Na-F and Na-P PPDF at 6.1 and $6.9 \AA$, respectively (Figure $3 \mathrm{~B}$ ), suggesting that the anion coordinates to the $\mathrm{Na}^{+}$(diglyme) complex. The hexafluorophosphate ions also exhibit weak hydrogen bonding between the $\mathrm{F}$ on the anion and the $\mathrm{H}$ of the diglyme with a bond distance of $2.5 \AA$ (Figure $3 \mathrm{C}$ ). Almost no intermolecular bonding is observed between diglyme molecules both with and without the salt present; only very weak hydrogen bonding is observed at $2.8 \AA$ (Figure 3D) slightly more pronounced for the $\mathrm{H}_{\mathrm{e}}$ compared to the $\mathrm{H}_{\mathrm{m}}$.

From the first peak in the PPDFs of the $\mathrm{Na}-\mathrm{O}$ and $\mathrm{Na}-\mathrm{F}$ pairs, the coordination number distribution was calculated (Figure 4A), showing that $82 \%$ of the sodium ions have no fluorine in the first coordination shell. Instead, the Na preferentially binds to diglymes, with most sodium ions having 5-6 oxygens in the first coordination shell (Figure 4A); no sodium was observed with 7 or more coordinating oxygens. The size of clusters was also examined, and is defined as the number of molecules and ions connected by a direct $\mathrm{Na}-\mathrm{O}$ bond (Figure $4 \mathrm{~B}$ ), with $\mathrm{n}=1$ being an isolated 

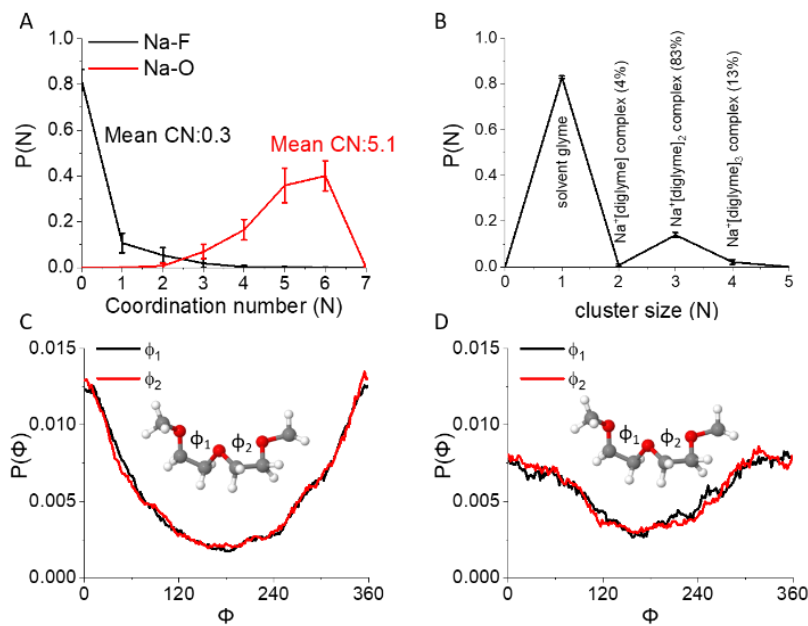

Figure 4: A) coordination number distribution of $\mathrm{F}$ and $\mathrm{O}$ around $\mathrm{Na}$ based on the first peak shown in the PPDFs (figure $3 A-B$ ). B) Cluster analysis of $\mathrm{Na}$ diglyme clusters, showing the size of clusters bound together by direct $\mathrm{Na}-\mathrm{O}$ bonds, the composition related to each size and the relative fractions disregarding the free diglyme molecules. Internal dihedral angles of the diglyme molecules in the presence of $\mathrm{Na}(\mathrm{C})$ and in the pure solvent (D).

diglyme molecule or sodium ion with no O-Na bond. A large portion of diglyme molecules do not coordinate directly to a sodium ion. The larger clusters all have to contain at least one sodium and one diglyme with the most common of these being cluster sizes with $n=3$, indicating that the most common clusters are $\mathrm{Na}^{+}$(diglyme) ${ }_{2}$ complexes, consistent with the large number of 5 and 6 coordinated sodium (Figure 4A). If the free diglyme molecules are removed from the population, $83 \%$ of sodium is bound in these complexes. A small fraction of the $\mathrm{Na}^{+}$(diglyme) complexes also contain the anion as $\mathrm{Na}^{+}\left(\mathrm{PF}_{6}^{-}\right.$,diglyme $\left.{ }_{2}\right)$ as shown in Figure $5 \mathrm{C}$. Only $4 \%$ of the sodium ions are bound as $\mathrm{Na}^{+}$(diglyme) complexes, most of which contain 1-2 anions directly binding to the sodium ion, suggesting that only a very small fraction are forming aggregates with $>2$ ions directly bound together. Additionally, $13 \%$ of the sodium is bound by three diglymes, likely representing an intermediate state in a ligand exchange mechanism as shown in Figure 5B. The conformational structure of the diglyme can be assessed by the dihedral angles in the diglyme (Figures 4C-D). A strong preference for a $0 \% 360^{\circ}$ dihedral angle is observed for diglyme in the presence of $\mathrm{Na}$ (Figure 4C), indicating a stabilized synperiplanar O-C-C-O rotamer relative to the pure solvent (Figure 4D), similar to that observed for LiTFSI solvated in tetraglyme. ${ }^{[22]}$ The broad distribution observed for pure diglyme is consistent with spectroscopy studies showing a broad distribution of conformations. $^{[29]}$ In highly agglomerated systems ${ }^{[22]}$ no preference is observed as most of the molecules do not interact with the cation and the preference of the synperiplanar rotamer reflects the strong solvent-cation interaction. Combining the results shown in Figure 4, the $\mathrm{Na}^{+}$(diglyme) $)_{2}$ forms a distorted octahedral coordination complex with two diglymes providing 5-6 oxygens to form direct bonds with the sodium (examples shown in Figure 5A). However, the intramolecular O-O distance is too short to form a perfect octahedral complex as observed from the angular distribution (Figure S7), revealing a large amount of disorder in the first coordination shell. The $\mathrm{Na}^{+}$(diglyme) ${ }_{2}$ complex does match well with the description of the $\mathrm{Na}^{+}$(diglyme) ${ }_{2}$ complex intercalated into graphite. However, as shown in Figure 5 the complex does not necessarily adopt the mer-isomer suggested from DFT for intercalated $\mathrm{Na}^{+}$(diglyme) ${ }_{2}{ }^{[5 \mathrm{~b}]}$ and the lack of $\mathrm{C}-\mathrm{C}$ and $\mathrm{O}-\mathrm{O}$ medium range order correlation in the PPDF (Figure S5) suggest a mixture of fac and mer isomers as expected from a liquid.

Around $18 \%$ of the sodium does form CIP with the anion, as shown in Figure $5 \mathrm{C}$. Here the sodium ion binds to the anion either in the inner coordination sphere (Figure 5D, purple site) at the bi-

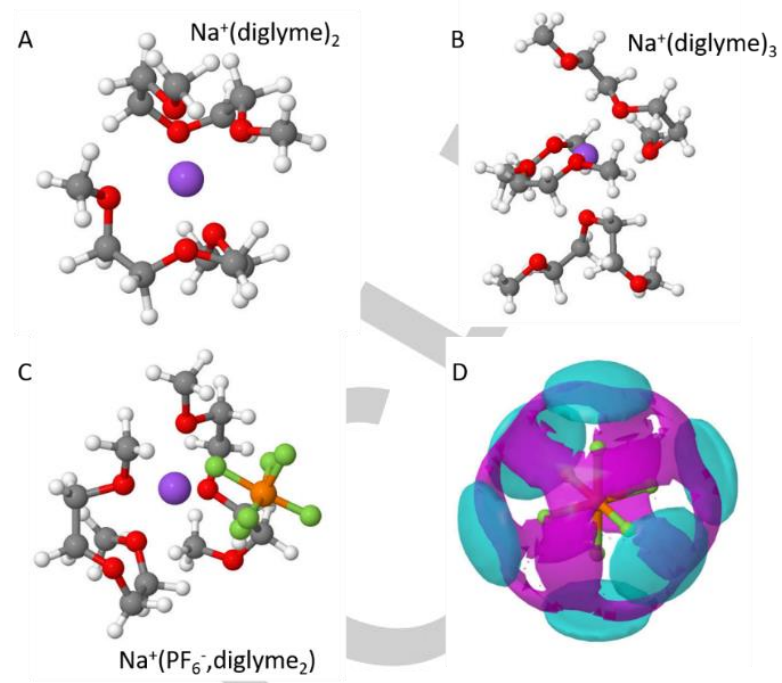

Figure 5: Example structures of the common solvation spheres for sodium of A) $\mathrm{Na}^{+}$(diglyme) $)_{2}$ and $\left.\mathrm{B}\right) \mathrm{Na}^{+}$(diglyme) $)_{3}$ and the less common $\mathrm{C}$ ) $\mathrm{Na}^{+}\left(\mathrm{PF}_{6}{ }^{-}\right.$, diglyme 2$)$. D) Spatial density function of sodium coordinating to $\mathrm{PF}_{6}$ - showing the inner (purple) and outer (light blue) coordination spheres observed from the double peak at $\sim 3 \AA$ in the Na-P PPDF (Figure 3B). The atoms are color-coded as follows, H: White, C: Grey, O: Red, F: Green, Na: Purple, P: Orange.

and tridentate sites of the $\mathrm{PF}_{6}$ anion, or the sodium ion can bind in the outer shell at the monodentate site (Figure 5D, light blue site).

The $\mathrm{Na}^{+}$(diglyme) $)_{2}$ complexes observed here are very similar to those observed for LiTFS|[22] in tetraglyme and $\mathrm{NaFSI}^{[21]}$ in triglyme, and the ion complexes which have previously been suggested to enable electrochemical intercalation into graphite. ${ }^{[3 a]}$ Neither $\mathrm{LiNO}_{3}$, LiTFSI ${ }^{[22]}$ or $\mathrm{NaOTf}{ }^{[19 \mathrm{c}]}$ exhibited a pronounced second coordination shell of the anion around the cation which we observed in this study with $\mathrm{NaPF}_{6}$ from the second peak in the Na-P PPDF (Figure 2b). However, a similar peak has been reported for $\mathrm{LiPF}_{6}$ in propylene carbonate consistent with the anion coordinating to the first solvation shell of the cation; this coordination may be a distinct feature of this anion. ${ }^{[18,30]}$ The high proportion of SSIPS may explain why the diglyme solution outperforms most other chain length glymes as an electrolyte solvent, ${ }^{[5 a]}$ since two diglymes contribute the exact number of oxygens necessary to fully solvate the sodium ion. This suggests that a large portion of the sodium is in the right conformation for intercalation at any given time. However, more extensive studies are required of the structure of different glyme lengths to fully elucidate the electrochemical intercalation behaviour.

The CIPs formed are not exclusively bidentate or tridentate as expected from the low energy of these complexes, ${ }^{[19 a, 19 b]}$ but are composed of a mixture of mono, bi and tridentate compounds as seen in figure $5 \mathrm{D}$.

\section{Conclusion}

Using neutron diffraction with EPSR we have shown that the most common solvation conformation of sodium in diglyme is $\mathrm{Na}^{+}$(diglyme) $)_{2}$, forming a stable complex with $\sim 83 \%$ of the sodium in this state. This state is also reported to be the most common state for $\mathrm{NaFSI}^{[21]}$ and LiTFSI ${ }^{[22]}$ salts in glymes. It also corresponds to the complex reported for electrochemical intercalation into graphite ${ }^{[5 b]}$ for SIB, suggesting that the solution structure may be paramount to which electrochemical reactions take place during intercalation, and could be used as a guide to optimize electrolyte properties. The $\mathrm{Na}^{+}$(diglyme) ${ }_{2}$ is associated with the anion in a distinct coordination shell at a distance of $\sim 6$ 
$\AA$, also observed for $\mathrm{LiPF}_{6}{ }^{[30]}$ suggesting that this may be a general feature of this anion. Little to no aggregation is observed, consistent with the excellent ionic conductivity of this ion pair in solution. ${ }^{[18]}$

\section{Experimental Section}

Materials: bis(2-methoxyethyl) ether or diglyme (99.5\%, Sigma Aldrich) and $\mathrm{NaPF}_{6}(98 \%$, Sigma Aldrich) were used as received. Pure diglyme, and a $1 \mathrm{M}$ solution of $\mathrm{NaPF}_{6}$ in diglyme, were placed in standard TiZr cans for neutron diffraction. Six different contrasts were used for each sample d0 (fully hydrogenated), d6 $\left(\mathrm{CH}_{3}\right.$ groups deuterated), d8 (- $\mathrm{CH}_{2}-\mathrm{CH}_{2}-$ groups deuterated) and $\mathrm{d} 14$ (fully deuterated). 1:1 ratio by volume $\mathrm{d} 0: \mathrm{d} 6$ and $\mathrm{d} 8: \mathrm{d} 14$ mixtures were also measured. The deuterated d6-diglymes were synthesised as follows: a solution of tetraethylene glycol $(5.0 \mathrm{~g}$, $47.1 \mathrm{mmol}, 1$ equiv.), iodomethane-d3 ( $9 \mathrm{~mL}, 141 \mathrm{mmol}, 3$ equiv.), $\mathrm{KOH}$ powder (29.1 g, $518 \mathrm{mmol}, 11$ equiv.) and tetrabutylammonium bromide $(3.04 \mathrm{~g}, 9.42 \mathrm{mmol}, 0.2$ equiv.) in THF $(200 \mathrm{~mL})$ was stirred at r.t. for 4 days under nitrogen. The reaction mixture was passed through a Celite bed, and the bed was washed with dichloromethane $(50 \mathrm{~mL})$. The filtrate was evaporated to give a pale yellow residue, which was purified by flash column chromatography using a solvent system of $100 \%$ ethyl acetate to give a colourless viscous liquid (1.35 g, 70\%). The d8- and d14-diglymes were made using an equivalent procedure. Example NMR and GC spectra are shown in Figures S1-3.

Neutron diffraction: The TiZr cans were placed in the auto sampler at the SANDALS ${ }^{[31]}$ instrument at ISIS (Harwell, UK) and each sample was measured for 8 hours. Data reduction was done in Gudrun ${ }^{[32]}$ and data analysis was performed in EPSR25. ${ }^{[25]}$ EPSR starting conditions can be found in Tables S1-3. The density of the liquids was determined using an oscillating U-tube densitymeter.

\section{Acknowledgements}

We would like to thank ISIS for beam time allocation under the experiment code RB1820487 and Dr. Sarah Youngs, Dr. James Tellam and Dr. Jack Hayden at the ISIS deuteration facility for providing the deuterated diglyme molecules. A.J.D. and M.M.T. would like to acknowledge the Engineering and Physical Sciences Research Council for support (grant EP/R021554/1).

Keywords: diglyme • sodium ion batteries EPSR $\cdot$ Neutron

\section{References}

[1] T. Takashi, Y. Kazuki, H. Takeshi, T. Mizuho, N. Megumi, K. Yuichi, T. Naoki, D. Kaoru, W. Masayoshi, Chem. Lett. 2010, 39, 753-755.

[2] K. P. Lee, L. A. Kinney, R. Valentine, Toxicology 1989, 59, 239-258.

[3] aB. Jache, J. O. Binder, T. Abe, P. Adelhelm, Phys. Chem. Chem. Phys. 2016, 18, 14299-14316; bT. Inose, D. Watanabe, H. Morimoto, S.-I. Tobishima, J. Power Sources 2006, 162, 1297-1303.

[4] C. K. Christensen, E. D. Bøjesen, D. R. Sørensen, J. H. Kristensen, J. K. Mathiesen, B. B. Iversen, D. B. Ravnsbæk, ACS Appl. Nano Mater. 2018, 1, 5071-5082. aM. Goktas, B. Akduman, P. Huang, A. Balducci, P. Adelhelm, J. Phys. Chem. C 2018, 122, 26816-26824; bM. Goktas, C. Bolli, E. J. Berg, P. Novák, K. Pollok, F.
Langenhorst, M. v. Roeder, O. Lenchuk, D. Mollenhauer, P. Adelhelm, Adv. Energy Mater. 2018, 8, 1702724.

[6] K. Westman, R. Dugas, P. Jankowski, W. Wieczorek, G. Gachot, M. Morcrette, E. Irisarri, A. Ponrouch, M. R. Palacín, J. M. Tarascon, P. Johansson, ACS Appl. Energy Mater. 2018, 1, 2671-2680.

[7] W. Zuo, R. Liu, G. F. Ortiz, S. Rubio, T. Chyrka, P. Lavela S. Zheng, J. L. Tirado, D. Wang, Y. Yang, Journal of Power Sources 2018, 400, 317-324.

[8] S. Terada, H. Susa, S. Tsuzuki, T. Mandai, K. Ueno, K. Dokko, M. Watanabe, J. Phys. Chem. C 2018, 122, 16589-16599.

[9] F. Xie, Z. Xu, A. C. S. Jensen, H. Au, Y. Lu, V. AraulloPeters, A. J. Drew, Y.-S. Hu, M.-M. Titirici, Adv Funct. Mater., 0, 1901072.

[10] F. Nacimiento, M. Cabello, G. F. Ortiz, R. Alcántara, P Lavela, J. L. Tirado, Dalton Trans. 2019, 48, 5417-5424.

[11] Y. Li, Y. Lu, P. Adelhelm, M.-M. Titirici, Y.-S. Hu, Chem. Soc. Rev. 2019

[12] I. Hasa, X. Dou, D. Buchholz, Y. Shao-Horn, J. Hassoun, S. Passerini, B. Scrosati, J. Power Sources 2016, 310, 26 31.

[13] aM. Cabello, T. Chyrka, R. Klee, M. J. Aragón, X. Bai, P. Lavela, G. M. Vasylchenko, R. Alcántara, J. L. Tirado, G. F. Ortiz, Journal of Power Sources 2017, 347, 127-135; bM. Cabello, X. Bai, T. Chyrka, G. F. Ortiz, P. Lavela, R. Alcántara, J. L. Tirado, Journal of The Electrochemical Society 2017, 164, A3804-A3813.

[14] T. Mandai, K. Dokko, M. Watanabe, Chem. Rec. 2019, 19, 708-722.

[15] aS.-D. Han, S.-H. Yun, O. Borodin, D. M. Seo, R. D. Sommer, V. G. Young, W. A. Henderson, J. Phys. Chem. C 2015, 119, 8492-8500; bS. Neander, J. Körnich, F. Olbrich, J. Organomet. Chem. 2002, 656, 89-96.

[16] T. Mandai, S. Tsuzuki, K. Ueno, K. Dokko, M. Watanabe, Phys. Chem. Chem. Phys. 2015, 17, 2838-2849.

[17] S. Tsuzuki, W. Shinoda, S. Seki, Y. Umebayashi, K. Yoshida, K. Dokko, M. Watanabe, ChemPhysChem 2013 14, 1993-2001.

[18] C. Geng, D. Buchholz, G.-T. Kim, D. V. Carvalho, H. Zhang, L. G. Chagas, S. Passerini, Small Methods 2019 3, 1800208 .

[19] aN. R. Dhumal, S. P. Gejii, J. Mol. Struct. 2008, 859, 8692; bN. R. Dhumal, S. P. Gejii, J. Phys. Chem. A 2006, 110, 219-227; cJ. Wahlers, K. D. Fulfer, D. P. Harding, D. G. Kuroda, R. Kumar, R. Jorn, J. Phys. Chem. C 2016, 120, 17949-17959; dG. Åvall, J. Mindemark, D. Brandell, P. Johansson, Adv. Energy Mater. 2018, 8, 1703036. aE. Jónsson, P. Johansson, Phys. Chem. Chem. Phys. 2012, 14, 10774-10779; bM. Okoshi, Y. Yamada, S. Komaba, A. Yamada, H. Nakai, J. Electrochem. Soc 2016, 164, A54-A60; cM. Okoshi, Y. Yamada, A. Yamada, H. Nakai, J. Electrochem. Soc. 2013, 160, A2160-A2165; dK. L. Browning, R. L. Sacci, G. M. Veith, J. Electrochem. Soc. 2017, 164, A580-A586.

[21] P. Geysens, V. S. Rangasamy, S. Thayumanasundaram, K. Robeyns, L. Van Meervelt, J.-P. Locquet, J. Fransaer, K. Binnemans, J. Phys. Chem. B 2018, 122, 275-289. T. Murphy, S. K. Callear, N. Yepuri, K. Shimizu, M. Watanabe, J. N. Canongia Lopes, T. Darwish, G. G. Warr, R. Atkin, Phys. Chem. Chem. Phys. 2016, 18, 1722417236.

[23] D. Morales, R. E. Ruther, J. Nanda, S. Greenbaum, Electrochim. Acta. 2019, 304, 239-245.

[24] C. M. Burba, R. Frech, J. Phys. Chem. B 2005, 109, 15161-15164

aA. K. Soper, Mol. Simul. 2012, 38, 1171-1185; bA. K. Soper, J. Phys. Condens Matter 2010, 22, 404210. aO. S. Hammond, K. J. Edler, D. T. Bowron, L. TorrenteMurciano, Nat. Commun. 2017, 8, 14150; bD. T. Bowron, M. Amboage, R. Boada, A. Freeman, S. Hayama, S. DíazMoreno, RSC Adv. 2013, 3, 17803-17812.

[27] aA. C. S. Jensen, S. Imberti, S. F. Parker, E. Schneck, Y. Politi, P. Fratzl, L. Bertinetti, W. J. E. M. Habraken, J. Phys. Chem. C 2018; bA. K. Soper, D. T. Bowron, Chem. 
Phys. Lett. 2017, 683, 529-535; cD. T. Bowron, Procedia Mater. Sci. 2014, 7, 38-52.

[28] aW. L. Jorgensen, D. S. Maxwell, J. Tirado-Rives, J. Am. Chem. Soc. 1996, 118, 11225-11236; bS. V.

Sambasivarao, O. Acevedo, J. Chem. Theory Comput. 2009, 5, 1038-1050.

[29] P. Johansson, J. Grondin, J.-C. Lassègues, The Journal of Physical Chemistry A 2010, 114, 10700-10705.

[30] Y. Kameda, Y. Umebayashi, M. Takeuchi, M. A. Wahab, S. Fukuda, S.-i. Ishiguro, M. Sasaki, Y. Amo, T. Usuki, J. Phys. Chem. B 2007, 111, 6104-6109.

[31] aC. Benmore, A. Soper, Council for the Central Lab. of the Research Councils (CLRC), 1998; bM. Dove, S. Gaertner, Z. Guo, H. Au, A. Drew, A. Jensen, S. Imberti, STFC ISIS Neutron and Muon Source, 2018.

[32] A. Soper, RAL Technical Report RAL-TR-2011-013 2011. 


\section{Entry for the Table of Contents}
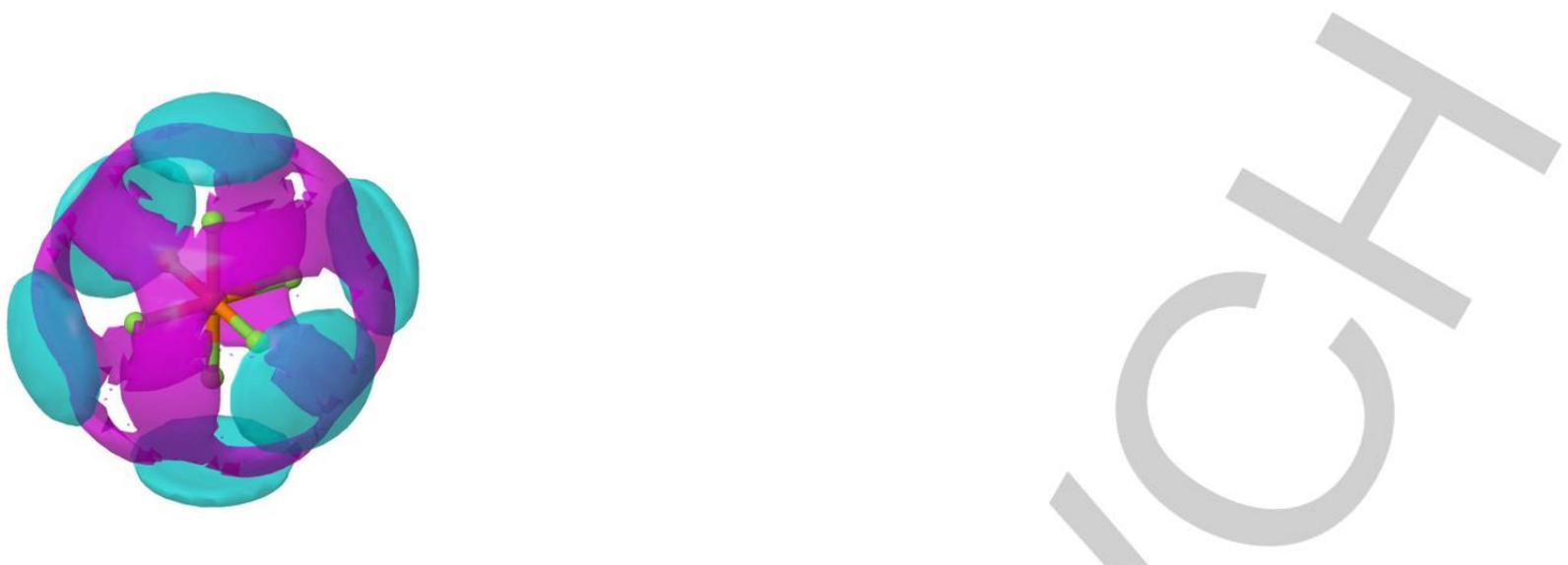

Diglyme is a promising electrolyte for sodium ion batteries technology and have shown good performance and low SEI formation with carbon anodes. Using total neutron scattering the local structure of the sodium in diglyme was observed and the diglyme molecules was found to physically separate the sodium cation from the anion by forming a $\mathrm{Na}^{+}(\text {Diglyme })_{2}{ }^{-}$complexes suggesting that these complexes may be the underlying reason for their high performance 